\title{
A DYNAMIC METHOD FOR MEASUREMENT OF MUSCLE TONUS IN MAN
}

By ABIGAIl E. SMITH, D. S. MARTIN, P. H. GARVEY AND W. O. FENN

(From the Department of Physiology and the Department of Medicine, School of Medicine and Dentistry, University of Rochester, Rochester, $N . Y$.)

(Received for publication November 14, 1929)

The problem of the quantitative measurement of the tonus of skeletal muscle in man has been attacked by a number of workers, and by nearly as many methods as workers. None of the methods has been entirely satisfactory, and many of them are too laborious for routine use. The method about to be described shares this fault. Its merits are that it furnishes a means of estimating tonus in absolute units (kilograms) in a moving limb.

The methods which have been used in the estimation of muscle tonus in man may be briefly reviewed. In 1896 Mosso stretched the gastrocnemius muscle by means of a lever and weights, and measured the amount of stretch. The "hardness" of muscle has been used as an index of tonus by a number of investigators. Noyons and Uexkull (1911), Mangold (1922), Gildemeister (1914), and Springer (1914), have used various devices for comparing the "hardness." Spiegel (1923) devised a method for measuring the tension in the quadriceps femoris by balancing the leg at different angles. It may be described as a static method. The tension is calculated in kilograms, allowance being made for the dead weight of the leg.

Since changes in tonus are displayed more characteristically in moving limbs than in stationary limbs we have sought for a dynamic method of measurement. Several such methods have been devised. Filimonoff (1925) describes an apparatus to record the changes of resistance to passive movement. No quantitative analysis was attempted. McKinley and Berkwitz (1928) have an ingenious device for measuring the rate of displacement of a passively moved limb. Tonus is expressed in terms of the angle through which the limb moves before its acceleration becomes 0 . The method has the advan- 
tage of simplicity in that estimation of the moment of inertia of the arm and other calculations are avoided, but the theoretical validity of this criterion of tonus may be called in question. Carmichael and Green (1928) have used a graphic method of recording the fall of a voluntarily relaxed forearm under the influence of gravity, using the length of time required for a fall through an angle of 80 degrees as an empirical index of the tonus of the biceps or triceps.

The method about to be described in this paper is essentially similar to that of Carmichael and Green, except that the leg is used instead of the arm, and that the displacement curve which records the rate of fall of the lower leg from the horizontal to the vertical position is analyzed mathematically to obtain values for tonus in absolute units. This method, like that of Spiegel, necessitates estimation of the weight, the location of the center of gravity, and the moment of inertia of the part. For this purpose we used the measurements made by Braune and Fischer (1889) on a limited number of cadavers which had been selected for their similarity in build and musculature. This necessarily introduces an error when subjects of widely different builds are used. However, the error remains the same for the same subject, and does not affect the shape of the tonus curve. The magnitude of the error thus introduced is estimated below.

\section{METHOD}

In order to record the fall of the leg the subject lies on his back with one leg strapped into a leather legging (see $L$, fig. 1 ) which is padded so as to fit snugly around the calf. The legging is supported by an oblong frame $(F)$ made of steel rods, the proximal end of which is left open to allow room for the knee joint. An additional strap is placed distally to support the ankle. The frame swings from a transverse axis at one end which is arranged to be continuous with the axis of the knee joint. On this axis a grooved wheel $(W)$ is fastened, which turns as the sling drops. A strong flexible wire passes over the groove in the wheel and is fastened to a small brass rider $(R)$ which is made to fit a rolled steel bar $(B)$ along which it slides smoothly. The other end of the rider is attached by a similar wire to a firmly anchored coiled spring $(S)$. This keeps the wires taut, and as the sling falls the coiled spring pulls the rider away from the wheel. The spring has been tested and proved to 
be able to pull the rider at a speed greatly in excess of the rate of drop of the sling, so that the rider will be compelled to follow the drop accurately and without lag. A pointer fastened to the rider writes the record of the fall on a fast moving smoked drum with horizontal axis. The drum is run at a constant speed by a motor which is placed on another table to prevent its vibrations from distorting the record.

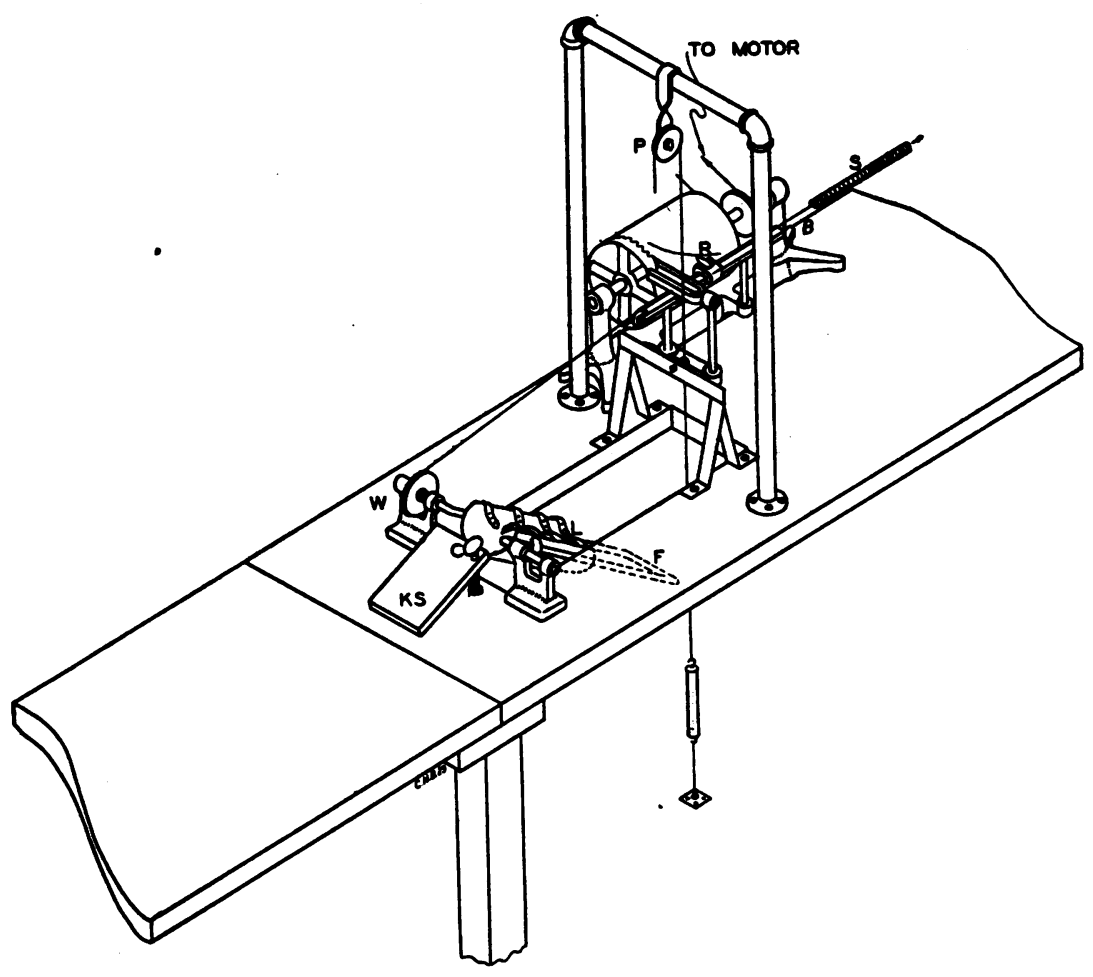

Fig. 1. Diagram of the Apparatus

For explanation see text

The sling is suspended horizontally by a catch at the distal end, from which it can be instantaneously released. A signal magnet in series with the releasing mechanism and an electrical tuning fork complete the recording apparatus.

Theoretically, a much lighter sling would be of advantage in the analysis of the records but the rigidity of the sling allows the recording 
apparatus to follow the drop of the leg more accurately and permits greater facility in effecting an instantaneous release of the leg. The frame is mounted on a large flat board with an oblong hole cut into it, large enough to permit the leg and sling to fall through it unhindered. This board is placed between two tables so that the leg has a free swing at all times. At the proximal end of the oblong hole is a small inclined board $(K S)$ serving as an adjustable knee support. The height of the support is regulated by a screw $(C)$ and is adjusted so as to take the weight of the thigh off the sling. There are wide limits of safety in this adjustment, both theoretically and practically and records have been taken with the board at different heights, with no perceptible change in the acceleration at any time during the fall.

\section{PROCEDURE}

The subject lies flat on his back on the long flat board and the leg is fastened into the sling in such a way that the condyles of the femur are opposite the axis of the sling. Inasmuch as the knee joint is not a pure hinge joint, and the axis of the joint changes position during the fall, this introduces some error which we have seen no means of avoiding. After the leg is strapped into place the patient is requested to relax completely. Pillows and padding are supplied as requested to insure absolute rest and comfort on the part of the subject. When the leg is released it drops like a pendulum. Before a record is taken several "drops" are carried out to acquaint the subject with the sensation of the drop and to remove any apprehensions which might cause extra voluntary muscle action. After the "practice" period, the drum is started and allowed to attain a constant speed and the vibrating tuning fork is touched to the drum as the leg is released. Many records can be taken in a few minutes. After the records are taken the torque in the horizontal position is measured by means of a spring balance and the distance from the femoral condyles to the sole of the foot is determined. The weight of the patient is also obtained.

\section{General outline of the method of analysis}

The fundamental law governing the fall of a compound pendulum like the leg in the sling is

$$
L=I A
$$


In words, a torque, $L$, produces an angular acceleration of $A$ in a body of moment inertia $I$. Now let the leg be fastened into the sling and be dropped so that it traces a record on the drum. $L$ and in consequence $A$ decreases as the leg falls. The drum tracing represents a graph in which the angular position, $\alpha$ is plotted as ordinates against time, $t$, as abscissae. If the radius of the grooved wheel $(W)$ is $r$, then $r \mathrm{cms}$. vertically on the drum tracing represents an angle between the sling and the horizontal of 1 radian or $360 / 2 \pi$ degrees. The slope $\frac{d \alpha}{d t}$ of this displacement curve at any point is the angular velocity of the fall. If the angular velocity is plotted in turn against time, the slope of the resulting curve, $\frac{d^{2} \alpha}{d t^{2}}$ represents the angular acceleration, $A$. If the sling is allowed to drop empty, without a leg, and if friction is negligible, then the value of $L$ calculated from $I$ and the observed angular acceleration, $A$, by equation (1) must be equal to the theoretical torque due to gravity $L_{o}$, calculated from the formula

$$
L_{g}=m . \text {.g.s. } \cos \alpha
$$

where $m$ is the weight of the sling at any distance $s$, from its axis of rotation, $g$ is the gravitational constant and $\alpha$ is the angle between the sling and the horizontal.

If friction is not negligible then $L_{g}-L$ is a measure of the magnitude of the friction. Similarly in the case of the leg, $L_{0}-L$, is a measure of all the forces both muscular and frictional, which resist the fall of the leg.

The graphical determination of the slope of the curve

The slope of the angular displacement curve (i.e., the slope of the drum record) at any point represents the angular velocity. The "mirror" method of obtaining slopes at points along irregular curves is recommended. By this method the mirror is set on edge, the flat surface being approximately at right angles to the graph at the point where it is desired to measure the slope. If the mirror is now rotated about this point as an axis it will be noted that there is one position at which the reflection of the graph in the mirror becomes continuous with the graph itself. In this position a line drawn along the edge of the mirror is perpendicular to the tangent to the graph at that point. The 
angular velocity of the leg at the point in question is then obtained by expressing in proper units (radians per second) the trigonometrical tangent of the angle between the edge of the mirror and the vertical. The mirror must of course reflect from its upper surface. By this method the slope can be determined with an error of less than 1 degree. It is recommended that the slope at each point be drawn twice by reversing the mirror, and facing it first in one direction and then the other. The results will check in most instances except where the slope is changing rapidly. In such cases the average of the two slopes is taken.

From these slopes a curve may be plotted with velocity as ordinates and time as abscissae. The slope of this smoothed curve, as determined again by the use of the mirror method, gives $\mathrm{A}$ the acceleration in radians per second per second.

\section{The fall of the empty sling and its moment of inertia}

In dropping a leg by the apparatus described and calculating the torque of the muscles, $L_{0}-L$, one is dealing with a system consisting of a leg plus a sling. The torque due to gravity, $L_{\theta}$, is the torque of the leg plus that of the sling. The torque of the sling is very simply determined by weighing it on a spring balance in the horizontal position $(\alpha=0)$. The torque of the leg must be estimated indirectly from the weight of the individual and the length of his leg as described later. Likewise the moment of inertia of the whole system is the sum of the moment of inertia of the leg plus that of the sling. Here again the former must be determined from the weight and dimensions of the individual. The moment of inertia of the sling however must be determined experimentally by recording the rate of fall of the empty sling.

For this purpose 10 records of the fall of the empty sling have been taken. At frequent intervals along these curves the angular velocities of fall have been determined by the mirror method. These velocities are plotted against time in figure 2, and a smooth curve is drawn through them. The scatter of the points is greater than the error in determining the velocities graphically. It must be concluded, therefore, either that the sling does not fall exactly the same way every time or that there is some mechanical error in the recording. 
One possible error of the latter sort is the shrinkage of the paper when shellaced. It is sometimes found for example that a base line traced on the revolving drum by a stationary pointer is not always exactly straight when the record is laid out flat, although the deviation is exceedingly small. In spite of such sources of error the points approx-

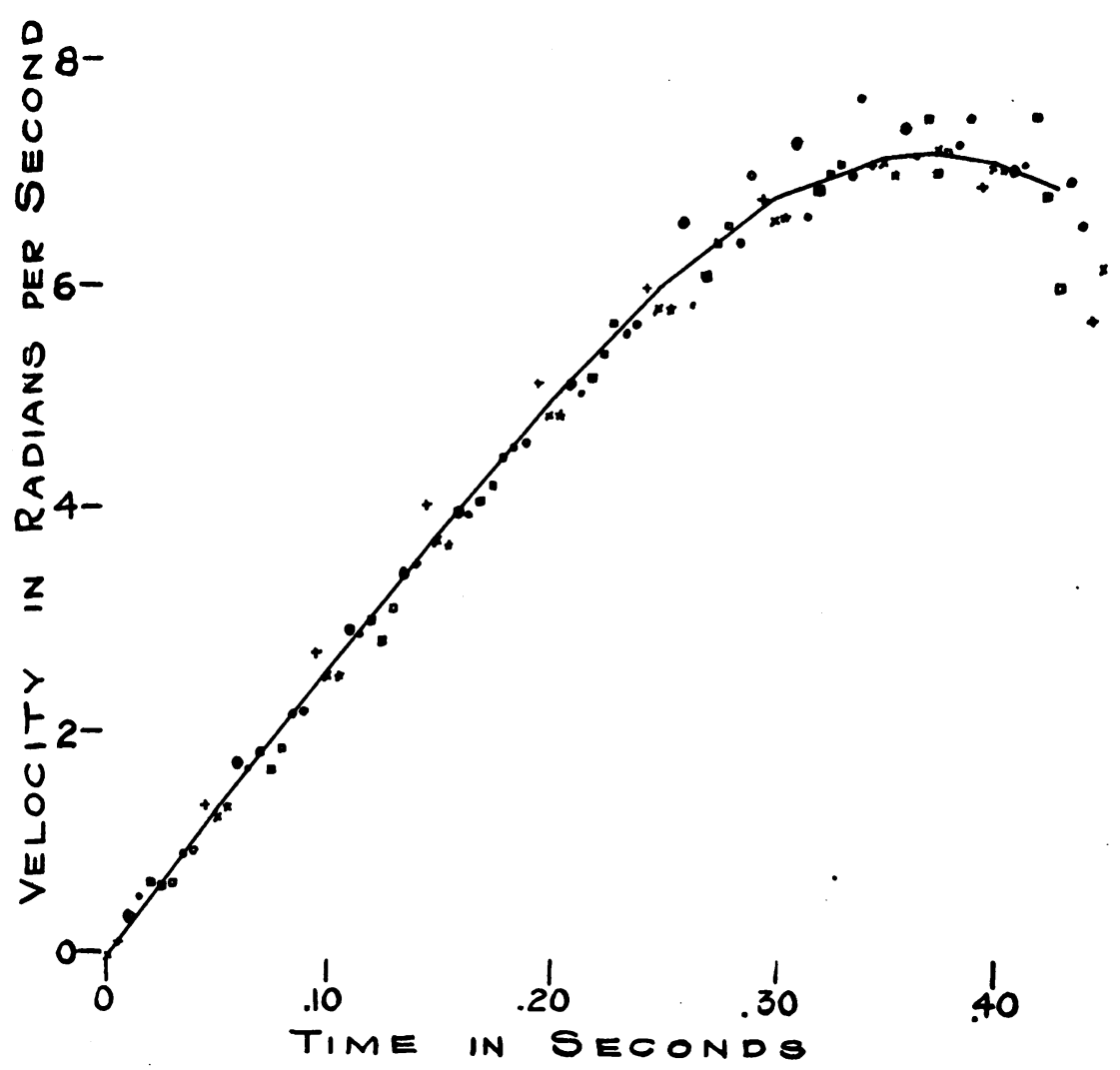

Fig. 2. Average Velocity Curve Determined from the ANalysis of 10 Drops of THE EMPTy SuING

imate very closely to the theoretical curve. To draw the theoretical velocity curve, the moment of inertia of the sling is first determined as described below. From the recorded displacement curve the mean position, $\alpha$, occupied at succeeding intervals of time is determined. The torque. $L_{a}$, for each of these positions is calculated from equation 
(2): Combining these values with the values of $I, A$ is determined by equation (1). Thus the slope of the velocity curve is known for each interval of time. The theoretical velocity curve can then be constructed by laying off the necessary slopes graphically for various values of $t$ and drawing a smooth curve which will have the proper slope at each point.

In practice, the moment of inertia of the sling is more readily determined from the initial slope of the velocity curve which is practically constant for the first 0.1 second. During this time the sling falls only 6.5 degrees and the resulting change in the torque, $L_{\theta}$, which varies with the cosine of the angle $\alpha$ is only from 1.00 m.g.s. to 0.994 m.g.s. If therefore the initial torque is determined by weighing the sling in the horizontal position, and if this is divided by the initial acceleration of the velocity curve, the quotient is the moment of inertia. The average figure so determined is $2.15 \times 10^{-6} \mathrm{gm} . \mathrm{cm} .^{2}$ and this figure has been used in our calculations. It may be mentioned here that the spring fastened to the recorder exerts a torque pulling in the same direction as gravity. This torque was found to be $4 \times 10^{6}$ dyne. $\mathrm{cm}$. at $0^{\circ}$ and $3.5 \times 10^{6}$ dyne. $\mathrm{cm}$. at $90^{\circ}$. This torque must be added to that due to gravity alone which is m.g.s. $\cos \alpha$ and has a value of 51.2 $\times 10^{6}$ when $\alpha$ is $0^{\circ}$ and falls to 0 when $\alpha$ is $90^{\circ}$. The total torque therefore varies from $55.2 \times 10^{6}$ at $0^{\circ}$ to $3.5 \times 10^{6}$ dyne. cms. and the difference $51.7 \times 10^{6}$ equals the loss of potential energy in ergs during the fall. If friction is negligible this energy must all have been converted into kinetic energy. From the average velocity curve of the empty sling in figure 2 the angular velocity, $\omega$, at $90^{\circ}$ is found to be 7.15 radians per second, whence the kinetic energy is $\frac{I \omega^{2}}{2}=\frac{2.15}{2}(7.15)^{2}$ $\times 10^{6}=54.8 \times 10^{6} \mathrm{ergs} . \quad$ This is only a little larger than the expected value $51.7 \times 10^{5}$ and may be regarded as something of a check on the accuracy of the measurements.

\section{Torque and moment of inertia of the "dead leg"}

The torque of the dead leg at $0^{\circ}$, m.g.s., is calculated from the data of Braune and Fischer, taking the mass of the leg, $m$, as equal to onefifteenth of the body weight, and $s$ (the distance from the center of gravity to the knee joint) as 52 per cent of the length of the leg, $l$, meas- 
ured from the femoral condyles to the sole of the foot. The moment of inertia of the leg around the knee joint as axis, is determined by the formula, $I=m\left(k^{2}+s^{2}\right)$ where $k$ equals the radius of gyration of the leg around a transverse axis through its center of gravity. The value for $k$ is estimated as 33 per cent of the length of the leg by Braune and Fischer.

It is pertinent to inquire what error is introduced into the calculation by errors in the estimation of the moment of inertia, etc. Assuming in each case a 10 per cent error in estimating $m, k$, and $s$, it may be calculated that $L g$ may be in error by 15 per cent and $L$ by 18 per cent (assuming no error in measuring $A$ ). A value of $L g-L$ at $0^{\circ}$ which is less than 33 per cent $(15+18)$ of $L g$ is not therefore, significant. At $90^{\circ}$ however, $L g=0$ and hence a value of $L g-L$ must be correct to within 18 per cent of the error in $L$ alone. The fact that the values actually obtained for $L g-L$ at $0^{\circ}$ have been so near zero and generally slightly positive (indicative of slight extensor tone), when the relatively large error might well have given them a negative value, serves to give us some confidence in the calculations. The value of $L$ at $0^{\circ}$, (i.e., the torque of the leg plus sling in the horizontal position) may also be measured directly by a spring balance and this figure also agrees fairly well with that calculated from Braune and Fischer's data (i.e., $L g$ ) confirming again the reasonable adequacy of the calculations. However that may be, the variations in $L g-L$ met with in pathological cases are so large compared to the normal that in spite of errors of this type the method yields valuable information. On account of these difficulties it might be thought better to assume $L g-L=0$ at the beginning of the drop (i.e., no muscle tension) and then measure $L g$ directly with a spring balance before releasing the leg and calculate the moment of inertia of the leg plus sling from the initial acceleration. In practice however, the initial slope is rather indefinite and we have therefore chosen to rely on the statistical data, checking it in each case by a direct initial measurement of $L$ by a spring balance.

\section{The muscle torque}

It should be emphasized that $L g-L$ represents the algebraic sum of all the forces other than gravity which act upon the leg during the fall, a positive value indicating a retardation or an extensor torque. 
It includes therefore, the difference between the pull of the extensor and flexor muscles, skin tension and friction, joint friction, muscle friction and apparatus friction. Since presumably positive values are chiefly due to tone in the extensor muscles which are most extended when the subject lies on his back, it seems justifiable to express $L g-L$ as if it were a tension exerted by the quadriceps muscle alone and the other factors were negligible. This is done by dividing the values for $L g-L$ by a factor $h g$. where $h$ is the distance from the insertion of the quadriceps tendon on the patella to the axis of the knee joint (i.e., the moment of the force) and $g$ is the gravitational constant. We have used the following values for $h$ at different angles as determined by Spiegel (1923) from measurements of cadavers:

\begin{tabular}{cc} 
angle of fall & \multicolumn{1}{c}{$c$} \\
$0-15$ & $c m$. \\
$15-25$ & 4.45 \\
$25-35$ & 4.40 \\
$35-45$ & 4.30 \\
$45-55$ & 4.20 \\
$55-65$ & 4.10 \\
$65-75$ & 4.00 \\
$75-90$ & 3.98 \\
& 3.80
\end{tabular}

Figure 3 will help to clarify the relations between the various forces which can act upon the leg during the fall. Angles are plotted horizontally and torques vertically in such a way that an extensor torque is above while a flexor torque or a gravity torque is below the $X$ axis. $L g$ is the calculated pull of gravity, becoming 0 at 90 degrees. $L$ is the observed torque as calculated from the acceleration. As the angle increases $L$ usually assumes "extensor" values, i.e., the acceleration downward becomes negative. The point of maximum velocity is reached where $L$ crosses the $X$ axis, and thereafter the velocity decreases. The height of the shaded area between $L g$ and $L$ represents the pull of the muscles; in this case it always has positive or "extensor" values and might be properly replotted above the $X$-axis. As an indication of the relative magnitudes involved, the maximum torque which can be exerted by the muscles in both flexor and extensor muscles has been determined roughly by direct measurement with a spring balance and is plotted in the diagram as broken lines. The whole figure is really a combined length-tension diagram of the flexor 


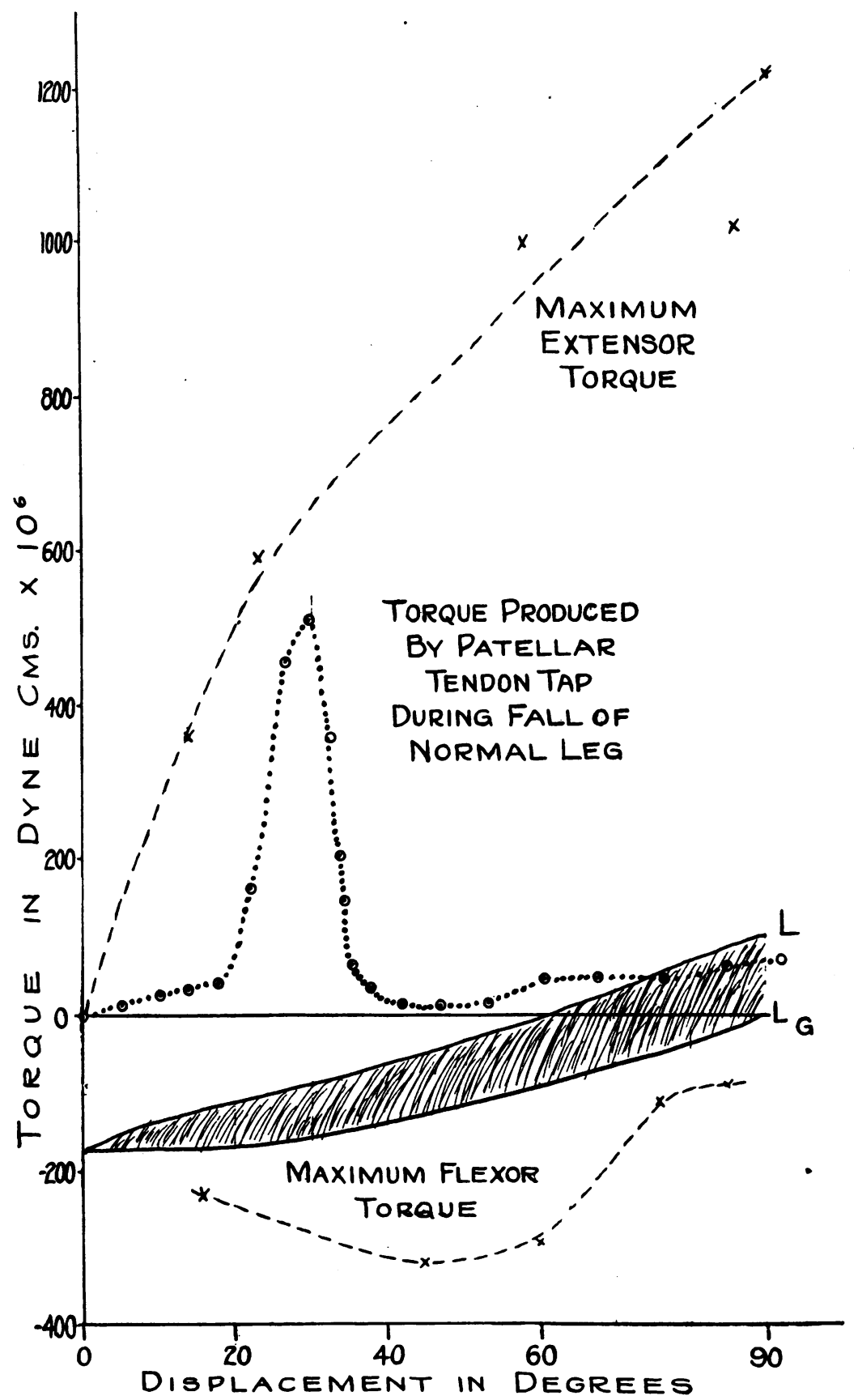

Fig. 3. Extensor Torque Plotted Upwards and Flexor Torque Plotted Downwards Against the ANgle

$L_{o}$ represents the flexor torque due to gravity: $L$ represents the observed torque in the same individual. The shaded area represents the work done by "extensor" forces in delaying the fall of the leg. The dotted line shows the extensor torque developed during the fall of a leg when the patellar tendon was simultaneously tapped. 
and extensor muscles and the area represents energy. To show further the magnitude of the tensions which may be developed by reflexes taking place during the drop, the values of $L g-L$ have been plotted from a case where the patellar tendon was tapped with a reflex hammer just as the leg was released, thus superimposing a knee jerk on the fall of the leg. The torque developed in this case was considerable (equivalent to $120 \mathrm{kgm}$. tension in the quadriceps) but less than the maximum possible at that angle. Presumably less than all the fibers in the muscle were stimulated.

If the calculated muscle torque $L g-L$ is plotted against the angle (as in figure 3 , shaded area) the result is in fact a tension-length curve

TABLE 1

Comparison of energy loss and area under T-l curve in four subjects

\begin{tabular}{|c|c|c|c|c|c|c|}
\hline & (1) & (2) & (3) & (4) & (5) & (6) \\
\hline \multirow[t]{2}{*}{ Subject } & M.g.s. & at $\stackrel{\omega}{90^{\circ}}$ & $\begin{array}{c}\frac{\mathrm{I} \omega^{2}}{2} \\
\text { at } 90^{\circ}\end{array}$ & $(1)-(3)$ & $\begin{array}{c}\text { Area of } \\
\text { T-l curve }\end{array}$ & Error \\
\hline & kgm. M. & $\begin{array}{c}\text { radians } \\
\text { per second }\end{array}$ & kgm.M. & kgm.M. & kgm.M. & per cent \\
\hline W. L.. & 1.47 & 5.02 & 0.68 & 0.79 & 0.76 & 3.9 \\
\hline P. H. G... & 1.78 & 4.70 & 0.75 & 1.03 & 0.97 & 6.2 \\
\hline P. H. G.... & 1.78 & 3.66 & 0.45 & 1.33 & 1.31 & 1.5 \\
\hline P. H. G...... & 1.78 & 3.55 & 0.43 & 1.35 & 1.32 & 2.3 \\
\hline
\end{tabular}

the area under which represents energy; in particular it represents the work done by the muscles (and friction) in retarding the fall. This area measured from $0^{\circ}$ to $90^{\circ}$ must represent the potential energy degraded into heat during the fall or the difference between the initial potential energy at $0^{\circ}$, m.g.s., and the kinetic energy, $\frac{I \omega^{2}}{2}$ at $90^{\circ}$ where the potential energy is 0 . To check further the accuracy of the analyses of the records the area under this tension-length curve was measured and compared with the energy loss in four cases chosen at random. The results are shown in table 1. In each case the energy loss as determined from the long and complicated analysis of the slope of the graph is equal to within 1 to 6 per cent of the value determined simply from the velocity of the leg at $90^{\circ}$. 


\section{Limitations of the analysis}

A word of caution may be introduced here as to the interpretation of slight waves in the velocity curve. Since the torque is calculated by multiplying the acceleration by the moment of inertia, and since the acceleration is markedly affected by a very slight wave in the veloc-

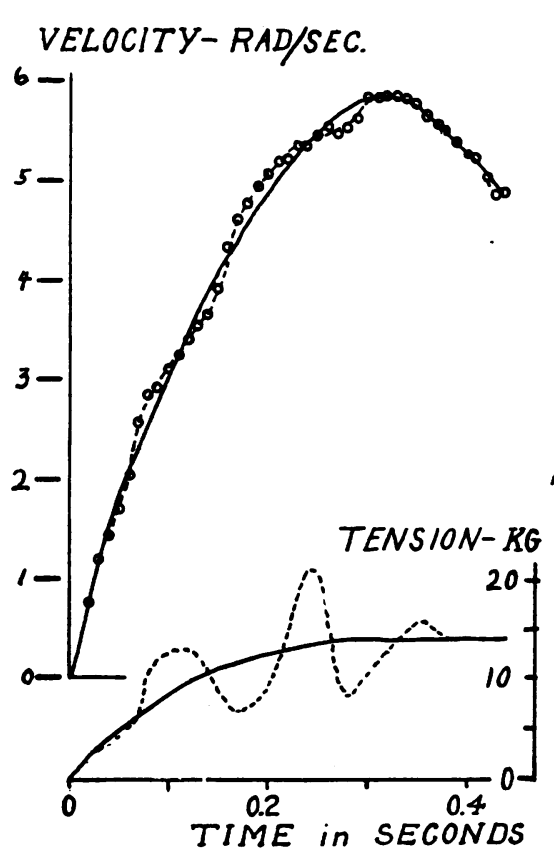

Fis. 4

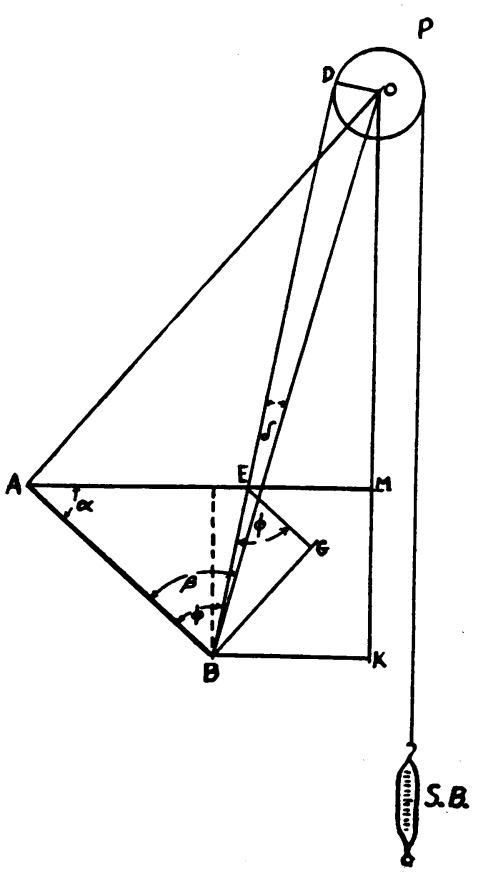

Fig. 5

Fig. 4. In the upper graph a smoothed curve is drawn through the actually observed values of the angular velocity. If the acceleration is determined from the slope of the smoothed velocity curve the continuous line in the lower graph results representing the calculated tension in the muscles. Otherwise the dotted curve results which is presumably erroneous.

Fig. 5. Diagram of the Forces Involved in the Determination of Tonus by the Static Method

ity curve, large variations can be introduced in the calculation of tension which might be interpreted as stretch reflexes. Figure 4, for example, shows the actual points obtained in the velocity curve measured on a normal individual. The solid line represents the smoothest curve that could be drawn through these points, and the dotted line 
follows the points faithfully and represents another possible interpretation. Below are shown the tension curves $\frac{L g-L}{h g}$. as calculated from each of the two upper curves. It seems hardly possible to trust the analysis so far as to believe that the tension actually varies according to the waving dotted line. It is at least more conservative to believe that these apparent rhythmic variations in velocity are within the limit of error of the method. We have not been able to determine the factors causing this error.

\section{Simultaneous determinations of static tonus}

This same apparatus lends itself admirably to the determination of tonus by the static method, by means of a slight modification of Spiegel's principle. It has the advantage that comparisons can be made between the static and dynamic method with the knee strapped into the same sling and without readjustment. It is a more rapid method than Spiegel's, easier to carry out, and quite accurate. An overhead pulley, $P$, about 3 feet above the board (see fig. 1) and approximately above the distal end of the sling is firmly anchored onto a pipe frame. A strong cord is then attached to the distal end of the sling (at $F$ ) passes up and over the pulley and then vertically downward where it is attached to a spring balance. The sling can then be lowered or raised quite easily and the tension in each position is read on the spring balance. The angle with the horizontal is determined by moving the drum a few centimeters by hand at each position and measuring the vertical distance from the base line. The calculations of the torque can then be carried out as follows (see fig. 5).

Let $A B$ represent the sling, swung from the axis $A ; F$, the force measured by the spring balance $(S B$.); and $O$, the center of pulley $P$. The force $F$ may be represented as a vector force $B E$ pulling along the line $B D$. A parallelogram of forces may be constructed with one arm $B G$ chosen perpendicular to the long axis of the sling $A B$ and representing the component of the force $B E$ which is perpendicular to the sling. $A B$, so that $B G \times A B=L$, the torque. But $B G=B E \sin \phi$ where $\phi$ = the angle $A B D=$ angle $B E G$.

Thence

$$
L=B E \times A B \sin \phi
$$

The calibration consists then in finding values of $A B \sin \phi$ for different positions of the sling. 
Let $\delta=$ the angle $O B D$ and $\beta=$ the angle $A B O$. It can easily be shown (according to the law of cosines) that in $\triangle O A B$

$$
\cos \beta=\frac{(O M+A B \sin \alpha)^{2}+(A M-A B \cos \alpha)^{2}+A B^{2}-A M^{2}-O M^{2}}{2 A B \sqrt{(O M+A B \sin \alpha)^{2}+(A M-A B \cos \alpha)^{2}}}
$$

and in $\triangle O B D$

$$
\sin \delta=\frac{O D}{\sqrt{(O M+A B \sin \alpha)^{2}+(A M-A B \cos \alpha)^{2}}}
$$

Knowing $\beta$ and $\delta, \phi$ is calculated by subtraction since $\phi=\beta-\delta$.

A calibration curve is then plotted with $A B \sin \phi$ as ordinates and $\alpha$ as abscissae. Thence after obtaining the records, the angle $\alpha$ is calculated from the height of the record on the drum and the corresponding values for $A B \sin \phi$ are read off from the calibration curve, and multiplied by the corresponding force observed on the spring balance, $F=B E$ to give the torque $L$ by equation (3). These values are corrected, taking into consideration the torque of the sling and the weight of the spring balance itself, and the tonus calculated from the dead leg torque, as described by Spiegel.

Unfortunately this improvement was not added until after the records of the pathological subjects were taken, so that we are not able to compare the relative values by the two methods in pathological cases. Measurements were, however, made in some cases by Spiegel's original method.

\section{EXPERIMENTAL}

Records have been taken and analyzed on 28 subjects, as follows:

12 normal subjects

8 cases of post-encephalitic pseudo-Parkinsonian syndrome

2 cases of multiple sclerosis

1 case of lateral sclerosis

1 case of hemorrhage into the internal capsule

1 case of spinal cord tumor

1 case of epilepsy

1 case of tabes dorsalis

1 case of pseudo-hypertrophic muscular dystrophy

We report in this paper only some preliminary observations on a few normal and a few pathological subjects. As an example of the method of analysis and the type of result obtained, figure 6 is presented showing displacement, velocity, and tension curves respectively of the following four cases: 

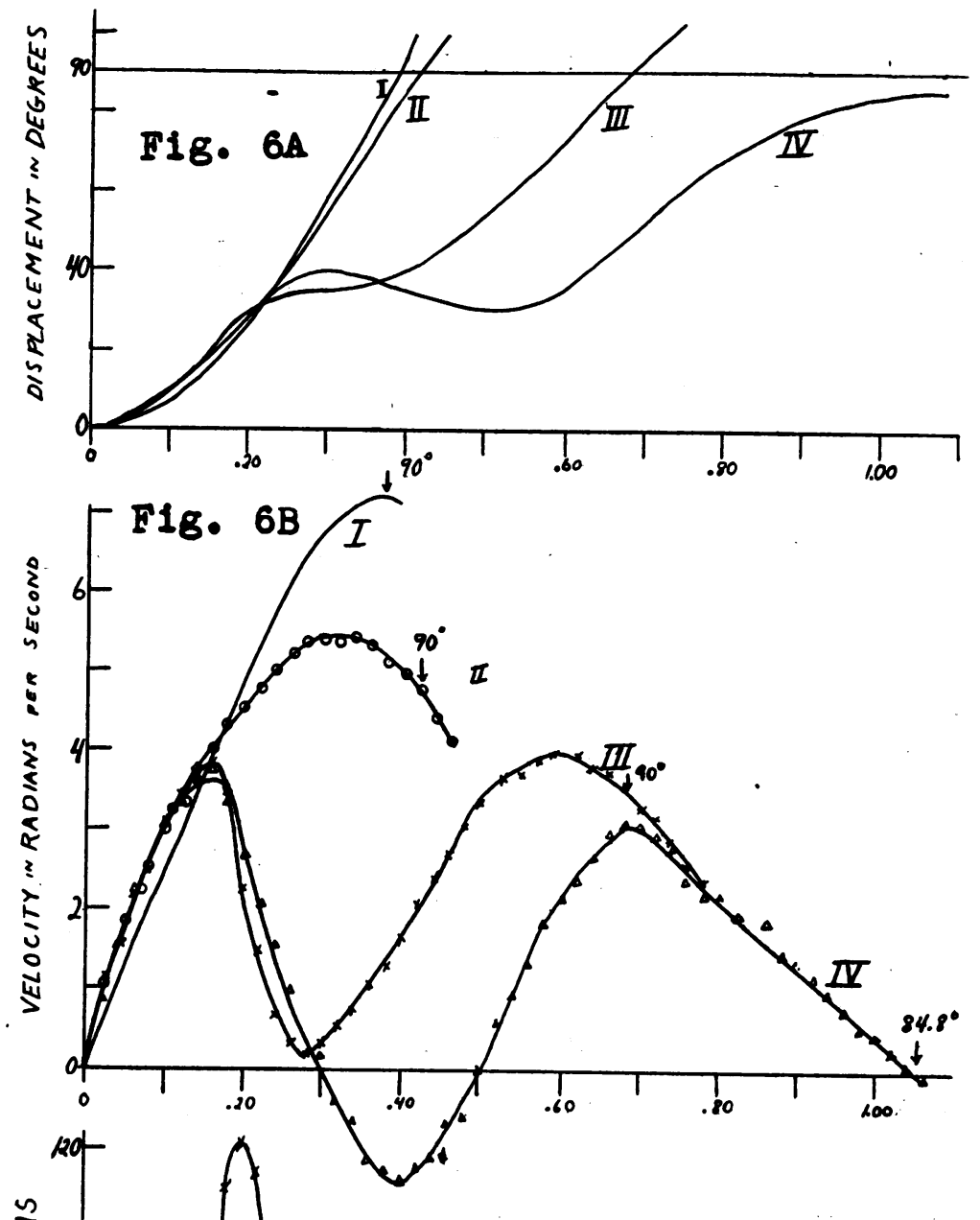

F1g. 6C

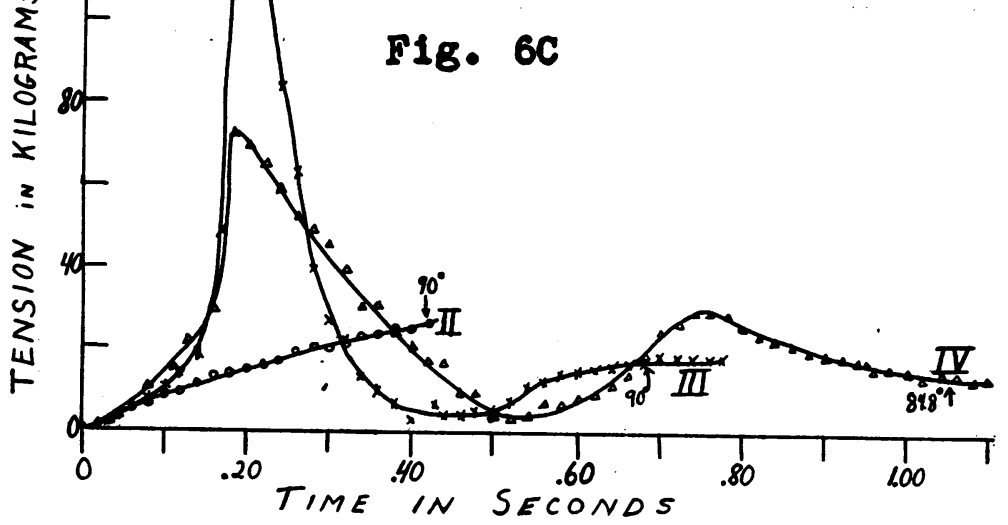

Fig. 6. Corresponding Displacement $(A)$, Angular Velocity $(B)$ and Tension (C) Curves Plotted Against Time for 4 Different Records

$I$, the empty sling: $I I$, a normal leg: $I I I$, a normal leg with an artificial knee jerk introduced, and $I V$, the leg of a spastic patient. 
I, empty sling

II, normal individual

III, same individual as II but with a knee kick produced during the fall by tapping the patellar tendon with a reflex hammer

IV, a patient with Parkinson's syndrome

It will be noted from the displacement curves of figure 4 that the times of fall to $90^{\circ}$ of the empty sling $(I)$ and the normal leg $(I I)$ are not as different as one might expect. Since the time for fall depends on $A=\frac{L}{I}=\frac{m . g . s .}{m\left(k^{2}+s^{2}\right)}$ and since when the leg is put on, both $k$ and $s$ are diminished (i.e., the pendulum is shorter) the value of $A$ becomes greater and the leg initially falls more rapidly than the empty sling. The subsequent retardation which causes the curves $(I$ and $I I)$ to cross is due to the increasing pull of the muscles.

It will also be noted that for the first 0.16 second, the leg of the patient with Parkinson's syndrome ( $I V$, fig. 6a) follows the same curve as the normal $(I I)$ and then a strong reflex contraction takes place which is strong enough to raise the leg and sling against gravity. In this case the leg reaches only an angle of $90^{\circ}$ with the horizontal, while a normal leg will swing beyond the vertical positions before being checked. The normal fall with the superimposed knee kick (III, fig. 6a) shows a strong reflex contraction followed by a rapid drop. The final velocity attained in this case is not, however, as great as if the fall had not been interrupted but is nevertheless greater than the final velocity in the case of the spastic leg $(I V)$.

Figure $6 \mathrm{~b}$ shows the velocity curves from the corresponding displacement curve of figure $6 \mathrm{a}$ by the application of the mirror method previously-described. An arrow on the curve indicates the nearest point to the $90^{\circ}$ position. Inspection of these curves reveals the following characteristic features: (1) the velocity of the empty sling at $90^{\circ}$ is much higher than in the case of any of the other drops; (2) that the leg reaches a maximum velocity before the angle of 90 degrees is reached (this is true in all of our records of falling legs, cf. curve $L$ in figure 3 which crosses the base line at the point of maximum velocity); (3) that in a strongly spastic leg, a negative velocity is attained (when the leg moves in the opposite direction).

Figure $6 \mathrm{c}$ shows the tension curves $(L g-L / h g$.) plotted against time, 
which correspond to the displacement and velocity curves of figures $6 \mathrm{a}$ and $6 \mathrm{~b}$. The values of $L$ used in calculating these tensions were derived graphically from the slopes of the velocity curves of figure $6 \mathrm{~b}$. It will be noted from these curves that the tension developed in the normal leg drop at 90 degrees is $27 \mathrm{kgm}$., while in the case of the same individual whose leg was checked by an artificially introduced knee jerk, the tension at 90 degrees is only $17 \mathrm{kgm}$. This lower value after the knee jerk interruption is due to the lower angular velocity attained. There is consequently less afferent stretch stimulus. Similarly in the case of the very spastic leg, at the angle of 84.8 degrees, the tension developed is only $13.8 \mathrm{kgm}$. Such figures show that the actual tensions developed in the muscles during the fall of the leg cannot be used arbitrarily as a quantitative measure of spasticity. Tensions at any given angle are comparable only when velocities of fall are equal.

\section{Tonus curves of normal individuals}

As might be expected, there is considerable variation in the muscle tone of different individuals, and in the same individual at different times. Voluntary changes in tonus no doubt come into play, which will considerably distort the record of involuntary response to stretch. Figure 7 (subject $I$ ) shows 6 length-tension curves of one individual taken at various times with the dynamic method (solid lines) as compared with the average of 2 records taken with the static method (dotted lines). This subject is a thinly built individual, of a rather quickly moving, nervous type. His tonus curves show rather wide variations in shape and magnitude. The static curve gives lower figures throughout since it is not being stimulated by stretching, but the general shape is the same. Theoretically the static and the dynamic curves should start at the same tension at $\mathbf{0}$ degrees. According to the discussions above the value of $L g-L$ at 0 degrees is significant only if greater than 30 per cent of $L g$ or $L$. In this individual $L g$ is 38 and the negative value is only 17.7 and is consequently within the error of method. At 90 degrees, however, $L g$ becomes zero and the error in estimating $L$ from $I A$ is only 15 per cent so that the maximum error of the difference $L g-L$ is only 15 per cent instead of over 100 per cent. The curves as a whole are therefore certainly significant.

Figure 8 (subject $I I$ ) shows four more curves on another normal 


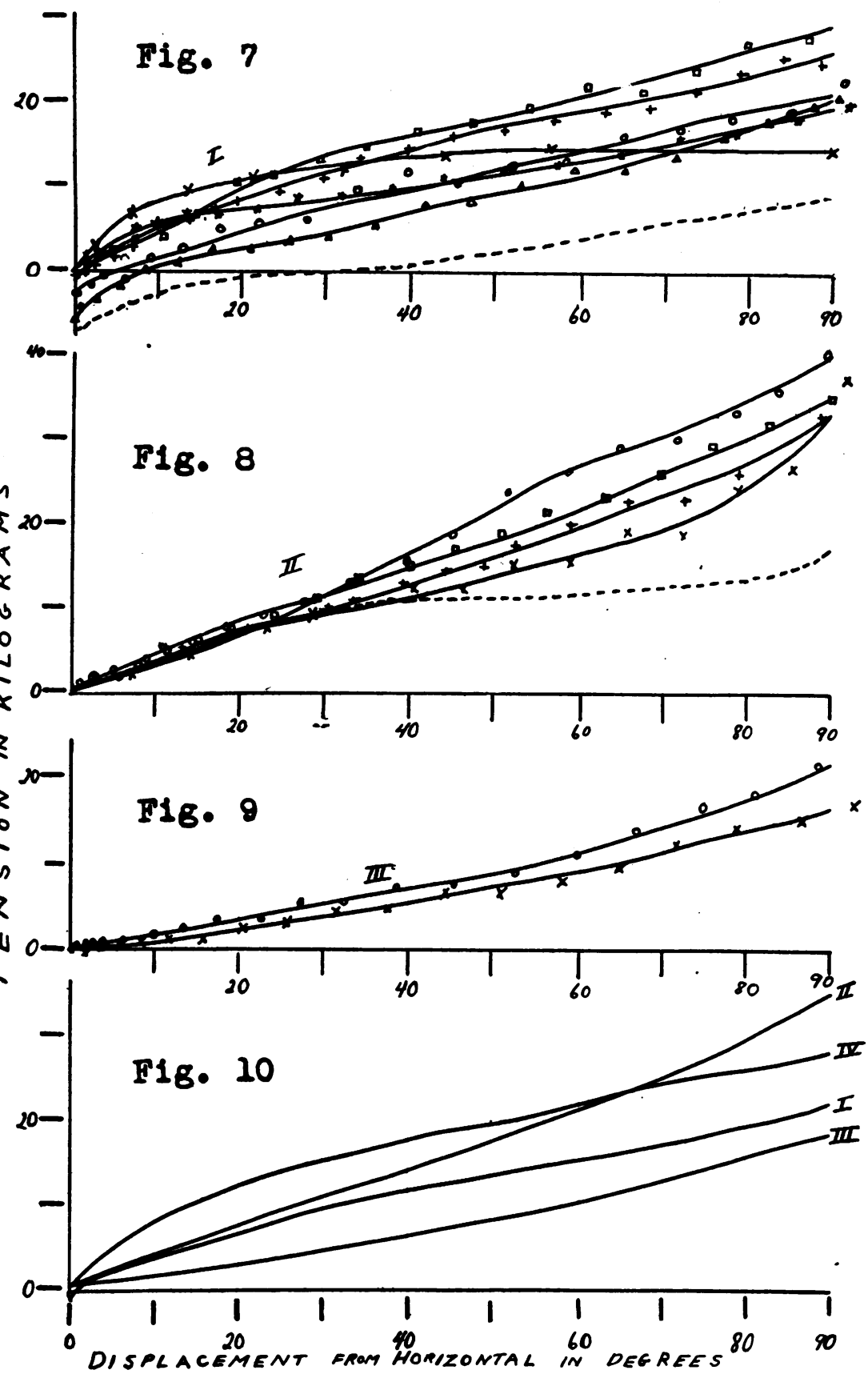

Figs. 7 to 10. Normat Muscle Tension Plotted Against Angle

Dotted lines represent static curves. Figures 7, 8, and 9 were taken from three different individuals. Figure 10 shows average curves from these three individuals together with one from a fourth individual (IV). 
subject, weighing 160 pounds, of rather stocky build, and phlegmatic temperament. All of his curves rise slowly and start from a point of zero initial tension. The static curves (dotted) in this subject rise with the dynamic curves and only leave them after the falling leg has attained a considerable velocity. The higher forces $(L g-L)$ found resisting the fall at 90 degrees in this case, may possible by due in part to friction and interference at the knee joint, since this subject has a considerable padding of fat around the joint.

Figure 9 (subject $I I I$ ) shows 2 tonus curves of a normal female. Figure 10 (subject $I V$ ) shows the average tonus curves of each of the individuals plus a single curve taken from a fourth normal individual.

Thus all these curves of normal individuals show in general a gradually increasing tension which reaches a maximum at $90^{\circ}$. This at least is the general trend of the curves. Minor variations and possible stretch reflexes like those illustrated in figure 4 have been omitted.

\section{Tonus curves of spastic legs}

In spastic legs, as shown in figure 6 the leg is often checked reflexly with such violence that it actually rises. This makes it very confusing to plot tension $(L g-L)$ against angle for the curve forms a loop. For this purpose it is therefore better to plot the tension against time as is done in figures 11 to 15 . The normal curves just described (fig. 10) are very short when plotted in this way as can be seen from figure 11 .

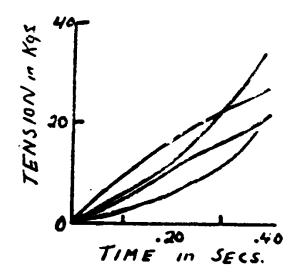

Fig. 11. Tension-Time Curves Corresponding to the Tension-Angle Curves SHOWN IN Figure 10

For comparison with figure 11 the tension-time curves of subject $V$ are drawn in figure 12. This subject was a thin, small individual diagnosed as post-encephalitic pseudo-Parkinson's syndrome. Clinically he did not appear very spastic but his graphs are far from normal.

Figure 13 (subject VI) shows two curves, one from each leg, of 


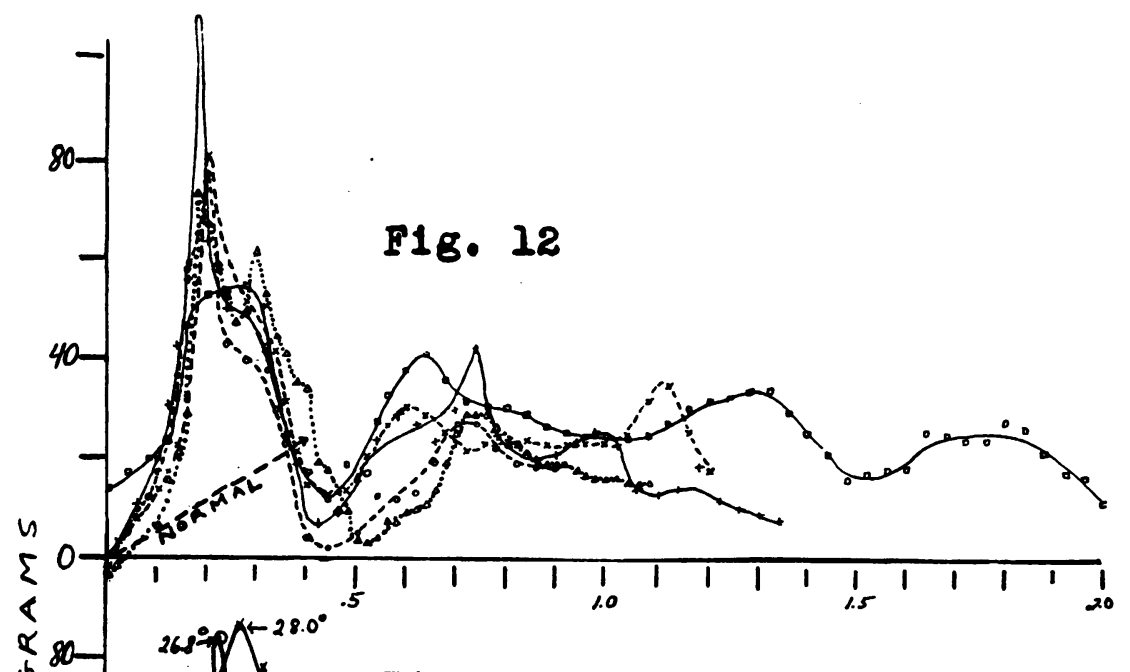

Fig. 13
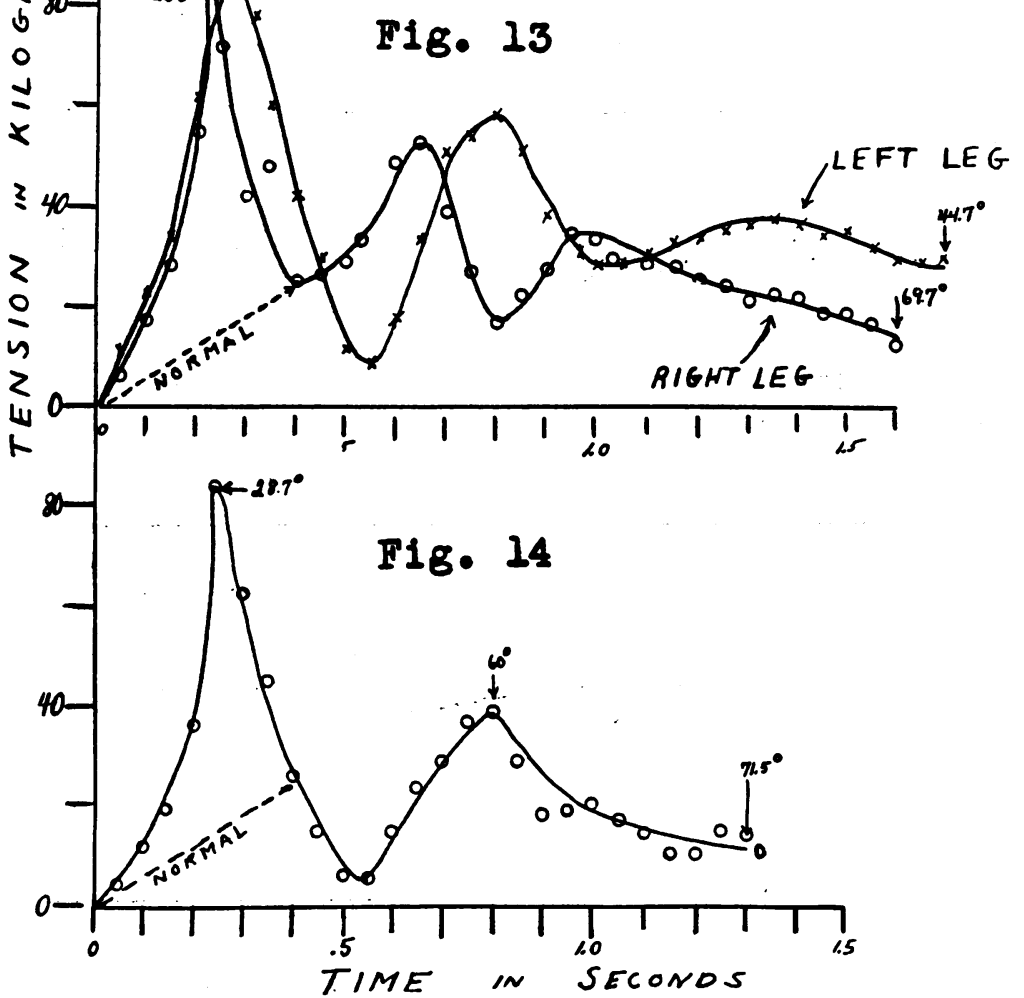

Figs. 12-14. Tension-Time Curves of Patients with Post-encephalitic Pseudo Parkinson's Syndrome 


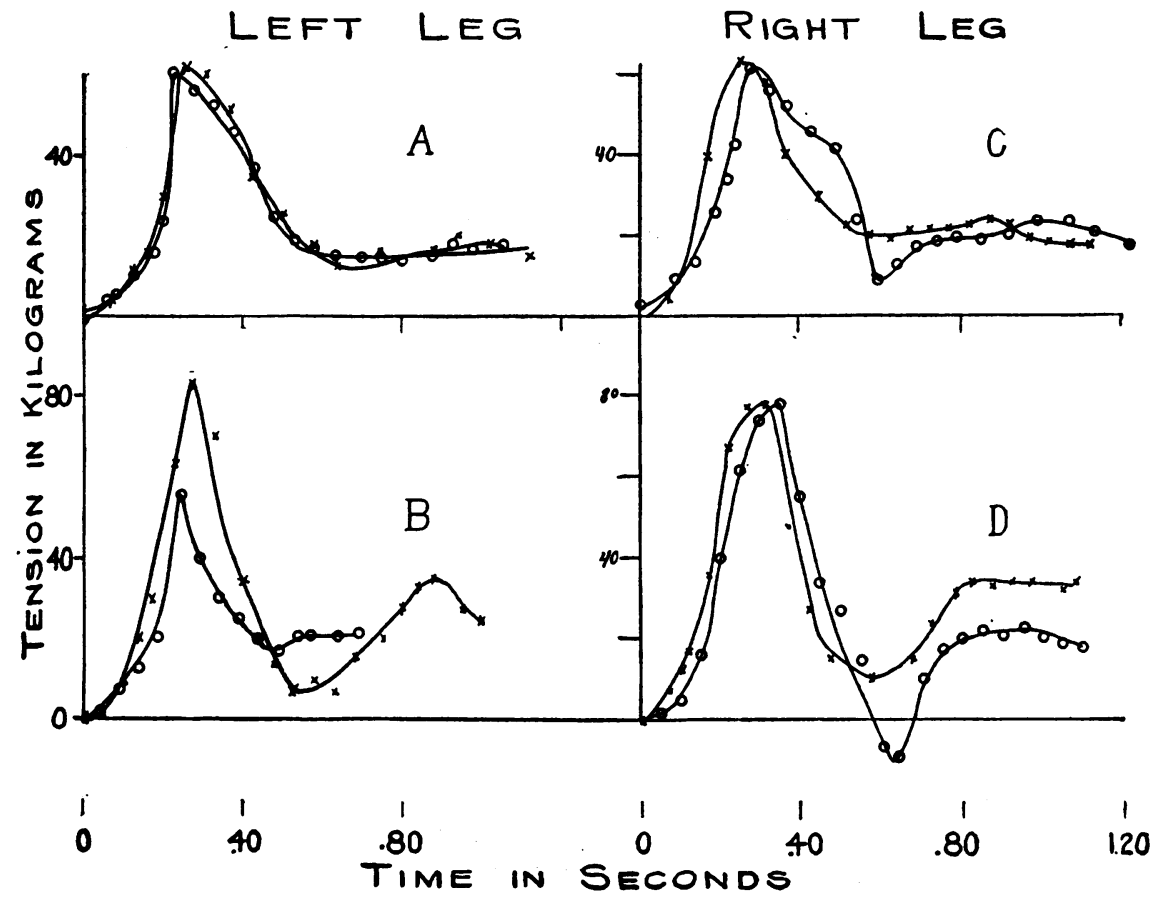

Fig. 15. Tension-Tme Curves of a Patient with Cord Tumor

$A$, left leg before operation; $B, 4$ weeks after operation; $C$, right leg before operation, $D, 4$ weeks after operation.

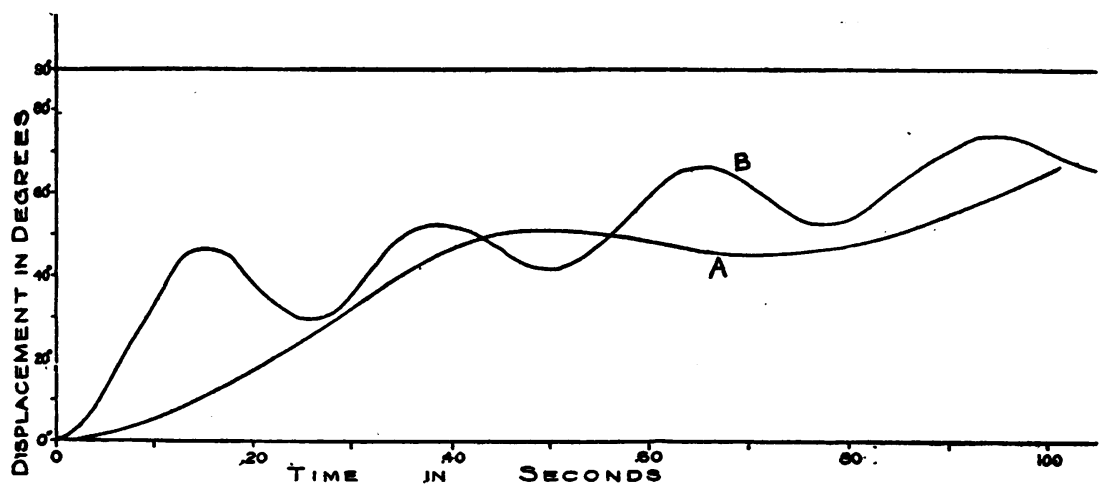

Fig. 16. Displacement-Time Curves of $A$, a Patient with lateral Sclerosis, and $B$, a Patient with Cerebral Hemorrhage 
a 41-year old male with knee jerks hyperactive, Babinski positive on right, negative on left, ankle clonus on left, and a hemiplegic gait. Clinically he was diagnosed as post-encephalitic pseudo-Parkinson's syndrome, with neurological evidence of bilateral pyramidal tract involvement.

Figure 14 (subject VII) shows one record on a 29-year old, undernourished, white male, clinically diagnosed as pseudo-Parkinson's syndrome. Reflexes were described as hyperactive. Marked increased resistance to passive movement was noted clinically.

Figure 15 (subject VIII) shows 8 curves of a 29-year old male $(62.8 \mathrm{kgm}$.) with a meningioma of the spinal cord. The patient was well developed and well nourished. Pyramidal signs were predominant. Two records were taken on each leg before operation for removal of the tumor and two more, four weeks after operation. At this time the records were still far from normal and the uniformity of the curves before and after operation is perhaps more striking than their differences. Previous to the operation the patient showed extreme spasticity and weakness of both legs, slightly more marked on the right. Tonus measurements by Spiegel's method showed also greatly increased tension, more marked on the right. Measurements made by Spiegel's method after the operation showed lessened tonus on both sides. Our dynamic method did not, however, show a marked change in the reflex response to a fall. It seems to measure in this case something which the static method misses.

All these curves from spastic legs show how such legs fall by jerks with alternating reflex contraction and inhibitions. The same phenomenon is equally evident in the displacement curves of figure 16 reproduced directly from the drum records. The subjects were two patients one with lateral sclerosis $(A)$ and one with cerebral hemorrhage $(B)$ the latter showing an especially beautiful series of waves during the fall.

Some interesting numerical comparisons between normal and spastic legs can be made as shown in table 2. In this table column 2 shows the area of the tension time curve, the average in normal cases being $456 \mathrm{kgm}$. seconds and in spastic legs $3329 \mathrm{kgm}$. seconds a seven fold increase. Column 3 shows the lowest position $(\alpha)$ to which the leg fell, many of the spastic legs failing to reach the vertical position (90 degrees) at all. The time taken to fall to this maximum angle is given 
TABLE 2

\begin{tabular}{|c|c|c|c|c|c|c|c|c|}
\hline \multicolumn{2}{|c|}{ (1) } & (2) & (3) & (4) & (5) & (6) & (7) & (8) \\
\hline \multicolumn{2}{|c|}{ Subject } & $\begin{array}{c}\text { Area under } \\
\text { curve }\end{array}$ & $\begin{array}{c}\text { Angle of } \\
\text { tall }\end{array}$ & $\underset{\text { fall }}{\text { Time of }}$ & $\begin{array}{l}\text { Average } \\
\text { tension }\end{array}$ & $\begin{array}{c}\text { Intial } \\
\text { potential } \\
\text { energy }\end{array}$ & $\begin{array}{c}\text { Energy } \\
\text { taken out } \\
\text { by muscles }\end{array}$ & $\begin{array}{l}\text { Energy } \\
\text { degraded } \\
\text { into heat }\end{array}$ \\
\hline \multicolumn{9}{|c|}{ Normal subjects } \\
\hline \multirow{7}{*}{ I } & & kgm. seconds & degrees & seconds & kgm. & joules & joules & per cent \\
\hline & 1 & 340 & 90 & 0.37 & 9.2 & 14.39 & 7.65 & 53.0 \\
\hline & 2 & 546 & 90 & 0.36 & 15.1 & 14.39 & 12.77 & 89.0 \\
\hline & 3 & 350 & 90 & 0.38 & 9.2 & 14.39 & 5.79 & 40.1 \\
\hline & 4 & 466 & 90 & 0.39 & 11.9 & 14.39 & 9.75 & 67.7 \\
\hline & 5 & 349 & 90 & 0.38 & 9.2 & 14.39 & 6.57 & 45.7 \\
\hline & 6 & 282 & 90 & 0.38 & 7.4 & 14.39 & 5.40 & 37.5 \\
\hline \multirow{4}{*}{ II } & 1 & 484 & 90 & 0.36 & 13.4 & 18.25 & 8.70 & 47.6 \\
\hline & 2 & 430 & 90 & 0.37 & 11.6 & 18.25 & 8.05 & 44.1 \\
\hline & 3 & 657 & 90 & 0.37 & 17.7 & 18.25 & 11.75 & 64.5 \\
\hline & 4 & 542 & 90 & 0.39 & 13.9 & 18.25 & 10.71 & 58.7 \\
\hline \multirow{2}{*}{ III } & 1 & 244 & 90 & 0.37 & 6.6 & 12.92 & 3.70 & 28.6 \\
\hline & 2 & 251 & 90 & 0.37 & 6.8 & 12.92 & 4.37 & 33.8 \\
\hline \multirow{2}{*}{ IV } & 1 & 820 & 90 & 0.46 & 17.8 & 17.64 & 13.20 & 75.0 \\
\hline & 2 & 620 & 90 & 0.44 & 14.1 & 17.64 & 10.12 & 57.5 \\
\hline \multicolumn{9}{|c|}{ Pathological subjects } \\
\hline \multirow{5}{*}{ V } & 1 & 2,595 & 84.6 & 1.04 & 25.0 & 13.77 & 12.50 & 100.0 \\
\hline & 2 & 3,165 & 69.8 & 1.16 & 27.3 & 13.77 & 9.24 & 100.0 \\
\hline & 3 & 3,613 & 85.2 & 1.74 & 20.8 & 13.77 & 5.24 & 100.0 \\
\hline & 4 & 5,500 & 85.0 & 2.46 & 22.4 & 13.77 & 12.56 & 100.0 \\
\hline & 5 & 3,280 & 84.7 & 1.34 & 24.5 & 13.77 & 11.41 & 100.0 \\
\hline \multirow{2}{*}{ VI } & 1 & 5,060 & 69.7 & 1.60 & 31.6 & 17.84 & 11.59 & 100.0 \\
\hline & 2 & 6,050 & 44.7 & 1.70 & 35.6 & 17.84 & 5.04 & 100.0 \\
\hline \multirow[t]{4}{*}{ VII } & 1 & 3,135 & 71.5 & 1.30 & 24.1 & 15.27 & 10.37 & 100.0 \\
\hline & 1 & 2,500 & 79.0 & 1.06 & 23.6 & 16.47 & 13.33 & 100.0 \\
\hline & 2 & 2,840 & 77.8 & 1.12 & 25.4 & 16.47 & 13.37 & 100.0 \\
\hline & 3 & 1,290 & 90.0 & 0.68 & 19.0 & 16.47 & 15.79 & 96.5 \\
\hline \multirow{5}{*}{ VIII } & 4 & 2,572 & 90.0 & 1.00 & 25.7 & 16.47 & 16.22 & 98.5 \\
\hline & 5 & 3,100 & 71.3 & 1.22 & 25.4 & 16.47 & 11.20 & 100.0 \\
\hline & 6 & 2,960 & 69.1 & 1.12 & 26.4 & 16.47 & 10.59 & 100.0 \\
\hline & 7 & 2,420 & 90.0 & 1.10 & 22.0 & 16.47 & 15.13 & 92.0 \\
\hline & 8 & 3,180 & 90.0 & 1.08 & 29.4 & 16.47 & 11.66 & 70.8 \\
\hline
\end{tabular}


in column 4. If the tension time of column 2 is divided by the time for the fall (column 4), the quotient gives a measure of the average tension during the fall and this figure is given in column 5. The average is $25.5 \mathrm{kgm}$. for the spastic compared to 11.7 for the normal legs. The initial torque, $L_{\theta}$, in the horizontal position represents the initial potential energy of the leg and sling (column 6). The difference between this and the kinetic energy (calculated from its angular velocity) of the system when it reaches the angle noted in column 3 at the end of its swing represents the work done by the system on the extensor muscles, joints, etc., or the energy degraded into heat during the fall (column 7). The last column shows that in the spastic legs nearly 100 per cent, while in the normal legs, only about 53 per cent of the potential energy lost was degraded into heat in the muscles, the remainder being in the form of kinetic energy at the end of the fall.

Other criteria for comparison of the tonus of muscles besides those suggested in table 2 might be used, as for example the angle at which the acceleration becomes zero (McKinley and Berkwitz). None of these criteria, however, expresses adequately the characteristic differences observed which may be summarized as follows.

1. The spastic leg is characterized by an exaggerated reflex response to stretching so that the resisting tension developed is greater than normal even though the speed of stretching is less.

2. The reflex resistance of the normal leg to the fall increases gradually and apparently smoothly (without at least any jerks which are greater than the experimental error of the method) while the spastic leg falls by very obvious jerks. The reflex response to stretch in the spastic leg is so great that the leg may rise against gravity.

No records of hypotonic legs have been included in this report but a limited number of observations on the tension curves of hypotonic subjects show curves similar to normal, but with less tension than normal. Reflexes are absent in these cases. Further discussion of such cases is reserved for a later time.

\section{SUMMARY}

A dynamic method of estimating the muscle tonus of man in absolute units is described.

The tonus curves of a few normals are compared with those of spastic subjects and discussed. 
The tonus curves of spastic subjects show alternate waves of tension during the fall of the leg.

\section{BIBLIOGRAPHY}

Braune, W., and Fischer, O., Abh. Sächs. Ges. Wiss., 1889, XV, No. VII, quoted in Oppenheimer u. Pincussen. Tabulae Biol., 1925, i, 86.

Carmichael, E. A., and Green, F. H. K., Quart. J. Med., 1928, xxii, 51. Parkinsonian Rigidity. A Clinical and Instrumental Study of the Effect of Stramonium, Hyoscine and Other Alkaloids.

Filimonoff, I. N., Ztschr. f. d. ges. Neurol. u. Psychiat., 1925, xcvi, 368. Klinische Beiträge zum Tonusproblem.

Gildemeister, M., Ztschr. f. Biol., 1914, lxiii, 183. Utber die sogenannte Härte tierischer Gewebe und ihre Messung.

Mangold, E., Pflüger's Arch., 1922, cxcvi, 200. Untersuchungen über Muskelhärte. I. Eine allgemein anwendbare Methode zur Physiologischen Härtebestimmung.

McKinley, J. C., and Berkwitz, N. J., Arch. Neurol. and Psychiat., 1928, xix, 1036. Quantitative Studies on Human Muscle Tonus. I. Description of methods.

Mosso, A., Arch. ital. de biol., 1896, xxv, 349. Description d'un myotonomètre pour etudies la tonicité des muscles chez l'homme.

Noyons, A., and von Uexküll, J., Ztschr. f. Biol., 1911, lvi, 139. Die Härte der Muskeln.

Spiegel, E. A., Ztschr. f. d. ges. Neurol. u. Psychiat., 1923, lxxxi, 517. Zur Physiologie und Pathologie des Skelettmuskeltonus.

Springer, R., Ztschr. f. Biol. 1914, lxiii, 200. Untersuchungen über die Resistenz (die sogenannte Härte) menschlicher Muskeln. 